\title{
Modelling of elastic-plastic behavior of cables
}

\author{
Oleksandr Shimanovsky* \\ V. Shimanovsky Ukrainian Institute of Steel Construction, 2/1, V. Shimanovsky str., Kyiv, 02125, \\ Ukraine
}

\begin{abstract}
The paper describes general calculation theory and elastic-plastic behavior of cables - bearing elements of suspension structural systems. It is mentioned that this theory is based almost on the same assumptions as the theory of cable calculation at behavior of material in elastic range, excluding additional supposition in the part of idealization of real dependence between stresses and deformations on account of difficulties with using the latter in actual structures design. For that reason, this dependence is replaced with a model in the form of analytic curve or, as it is accepted to say in this case, a diagram, which is built according to some simple mathematic rule, reflecting element behavior conditions and characteristics of its material. It is stated that four main models of material behavior are used in practice: elasticplastic, elastic-plastic with linear hardening, rigid-plastic and rigid-plastic with linear hardening. Conditions of occurrence of plastic deformations in all behavior stages of cable cross section are determined. Interrelations for geometrically and physically non-linear task of the cable at active loading are provided. Methods are given and limit values of loads acting on the cable are determined. Equations defining parameters of cable deflected mode in all deformation phases and conditions of changing phases of cable behavior are given.
\end{abstract}

\section{Initial assumptions}

It is known that the theory of deformation of plastic cables is based almost on the same assumptions as the theory of calculation of cables during behavior of material within elastic range. The only additional premise is connected with idealization of the stress-strain diagram of real materials, which in reality turns out to be quite complicated for direct application in design calculation. Therefore, in the plastic theory such an idealization is reduced not only to refusal to take into account the specific effects (creep, aftereffect and hysteresis) inherent in plastic deformation, but also to its maximum schematization. That is why the real relationship between stresses and deformations is replaced by some analytical curve plotted according to some simple mathematical law, reflecting the behavior conditions of the element and characteristics of its material. Occasionally, polygonal, parabolic and other power approximations are used [1-8]. In the majority of cases, this diagram is taken in the form of a broken line with two sections, one of which corresponds to elastic and the other to plastic ranges of behavior of material. In the development of what has been said, it is also necessary to point out that, more often than not, four basic models of behavior of material are used in practice: elastic-plastic, elastic-plastic with linear hardening, rigid-plastic and rigid-plastic with linear hardening (Fig. 1).

\footnotetext{
* Corresponding author: niipsk@,webber.kiev.ua
} 


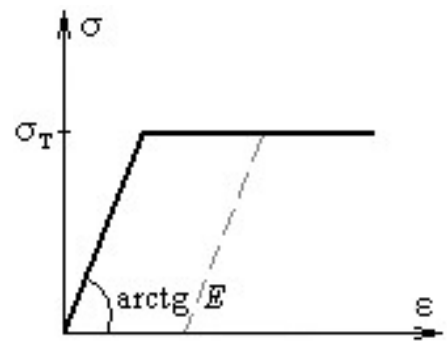

$a$

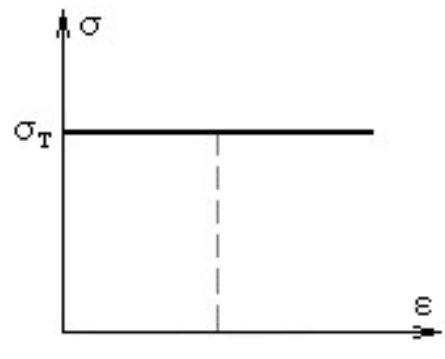

$c$

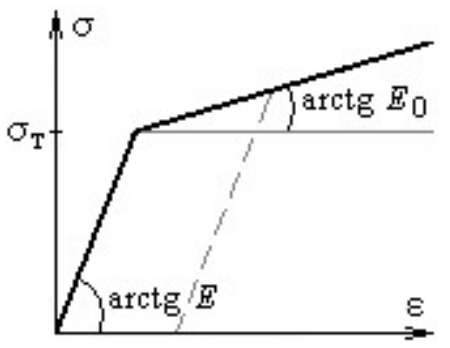

$b$

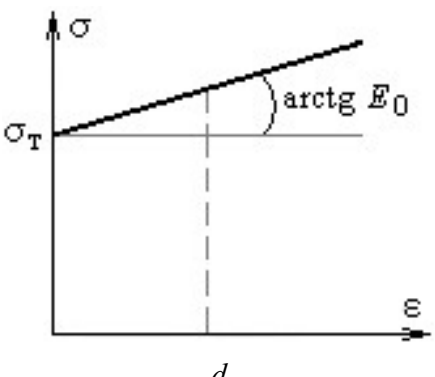

$d$

Fig. 1. Idealized stress-strain diagrams of the material: $a$ - elastic-plastic; $b$ - the same, with linear hardening; $c$ - rigid-plastic; $d$ - the same, with linear hardening.

Of the models mentioned, the most widely used model was the model of an ideal elasticplastic material (Prandtl material), the diagram of which is shown in Fig. 1a. Such an approximation as a whole quite satisfactorily reflects the behavior of many real materials and allows us to analyze behavior more fully in comparison with the elastic range of behavior and to reveal additional reserves of structural strength.

Subsequent stage in theory refinement is naturally related to the account of material hardening. It is understandable that, although hardening neglect causes a safety error and goes to strength reserve, this error is very significant for some materials and leads to creation of significant unaccounted (and often undesirable) safety reserve in the structure. And calculated justification of hardening is carried out by appropriate selection of the material behavior diagram. It is possible, for example, to form a diagram consisting of two inclined straight lines, the first of which corresponds to the elastic part of the behavior of material, and the other, usually having a smaller slope - to plastic one (Fig. 1b). In this context let us pay attention to such a very revealing aspect that an insignificant (at first glance) change in the diagram causes a radical restructuring of the theory in terms of complicating its calculated dependencies.

Now it is worth emphasizing that all the preceding reasoning related to the stress-strain curves approximation with respect to active load conditions, paraphrasing in other words, when the load acting on the structure increases continuously. Nevertheless, when the load is reduced, the behavior of the material that has received plastic deformation is described by a law that is fundamentally different from the loading law, namely: when unloading, the material behaves like an elastic body. In this case it is customary to extrude the curve of its (material's) deformation by an inclined straight line parallel to the section of the stretching diagram. This linear discharge continues until the stress reaches the yield strength of the opposite sign, after which the plastic flow begins again. Moreover, due to the difference in loading and unloading laws, after the force termination, the structure does not return to its original state, since some residual deformations appear in it. Note that the behavior of material during unloading is shown by the dashed lines in Fig. 1. 


\section{Cross-sectional behavior of cable made of ideal elastic-plastic material}

Let us consider the stress-strain state of an arbitrary cross section of an elastic-plastic cable (Fig. 2a). And first of all, let us establish the form of the relationship between the current value of cross-sectional force in the cable and its limit value, which attests to the appearance of a limiting state in the cross section. For this purpose, we design elementary forces acting in the plane of the cable cross section (the distribution diagram of which is shown in Fig. 2b) on the horizontal axis $z-z$ :

$$
\int_{F} \sigma d F=H
$$

where $\sigma$ is the normal stress; $F$ is the thread cross-sectional area; $H$ is the cross-sectional force.

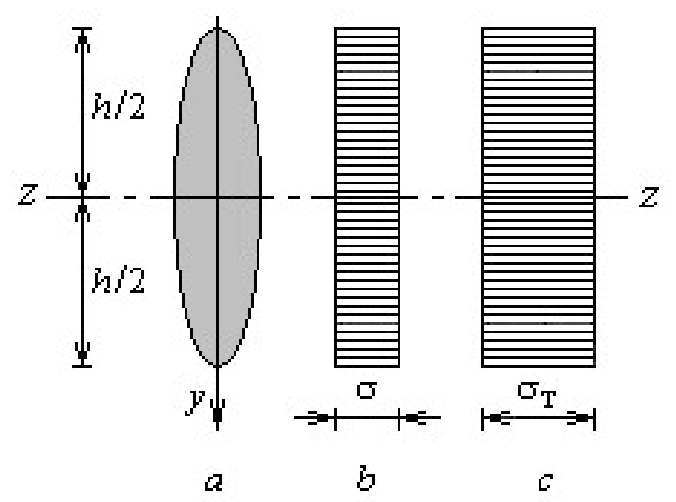

Fig. 2. Work of elastic-plastic cable cross section: $a$ - cable cross-section; $b$-stress diagram in elastic range of behavior; $c$ - stress diagram in limit state.

The normal stress $\sigma$ entering into expression (1) is a function of the relative elongation $\varepsilon$, the form of which is established in accordance with the accepted material tensile diagram. In the case of an ideal elastic-plastic material (Fig. 1a) for the first - elastic section of the diagram, this function can be represented in the form of:

$$
\sigma=E \varepsilon_{0} \quad \text { with } \quad \sigma<\sigma_{\mathrm{T}},
$$

where it is assumed that $E$ is the elasticity modulus; $\varepsilon_{0}$ is the relative elongation of the cable axis; $\sigma_{\mathrm{T}}$ is the yield stress.

If cross-sectional force acting in the cable does not cause yield stresses, then the cross section behaves in elastic range (Fig. $2 b$ ). Then, substituting condition (2) in the equilibrium equation (1) we arrive at the expression determining the relative elongation of the cable axis in this behavior range:

$$
\frac{E \varepsilon_{0}}{\sigma_{\mathrm{T}}}=\frac{H}{H_{\text {пр }}},
$$

in which the parameter of $H_{\text {пр }}=\sigma_{\mathrm{T}} F$ is the cable limiting cross-sectional force.

Returning to formulation of the problem, we note that the elastic range of the cross-section behavior will end when the cross-sectional force $H$ in the cable reaches its limit value $H_{\text {пр, }}$ and stresses reach the yield point $\sigma_{\mathrm{T}}$. After that, in accordance with the accepted diagram of the material stretching their magnitude will no longer increase (Fig. 2c). 
The condition for appearance of plastic deformations can be established by means of equilibrium equation (1), if we equate the value of the normal stress to the value of the yield stress $\sigma=\sigma_{\mathrm{T}}$. We shall have:

$$
\frac{H}{H_{\text {пр }}}-1=0 .
$$

Let us note that the obtained solution (4) has two principal aspects in applied respect, the physical meaning of which reveals the behavior essence of elastic-plastic cable. First, the appearance of plastic deformations in the cable cross section means the emergence of a limit state in it.

And the second, the most important: the limit state realization in any arbitrary cross section of elastic-plastic cable leads to propagation of plastic deformations along its entire length. Hence, in turn, it follows that the cable deformation process is carried out in the following sequence: for $\sigma<\sigma_{\mathrm{T}}$ all cable cross sections behave in the elastic range and for $\sigma=$ $\sigma_{\mathrm{T}}$ all of them pass to the limit state.

\section{The main dependencies for calculation of cable made of ideal elastic-plastic material}

Let us use the results obtained in the previous section for assembling equations of the elastic-plastic cable deformation theory (Fig. 3a). It is understood that the first deformation phase of elastic-plastic cable is elastic one. The stress diagram for this phase is shown in Fig. $3 b$. We recall that when the cable is working in this phase, the values of its stress-strain state parameters are similar to the corresponding parameters of an elastic cable.

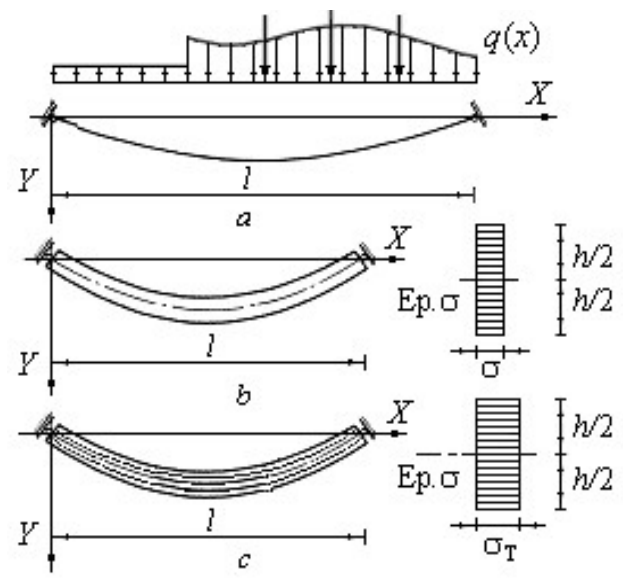

Fig. 3. Diagram of behavior of elastic-plastic cable: $a$ - design diagram of the cable; $b$ - elastic phase of behavior; $c$ - plastic phase of behavior.

It is easy to see that the elastic phase of behavior ends when, with increasing load, the cross-sectional force $H$ acting in the cable reaches its limit value $H_{\text {пр }}$. Then in the cable cross sections, first, condition (4) is satisfied, and secondly, the plastic deformations propagate along the entire length of the cable (Fig. $3 c$ ), thereby realizing the limit state in it.

In other words, the condition for termination of the first (elastic) phase of behavior of the cable is simultaneously a condition for beginning and end of the second phase - the plastic phase of its behavior, since plastic deformations cover the entire cable. Thus, the calculation of the cable having an ideal elastic-plastic stretching diagram of the material allows us to find only the magnitude of its ultimate load. And for this we can use the known solution for cables [9], assuming in it $H=H_{\text {пр. }}$ 
The corresponding calculation dependencies characterizing the termination condition for the first phase and beginning of the second phase of behavior, as well as determining the magnitude of ultimate load of the elastic-plastic cable are given in Table 1 for some of the most common types of loads.

Table 1. Calculation dependencies

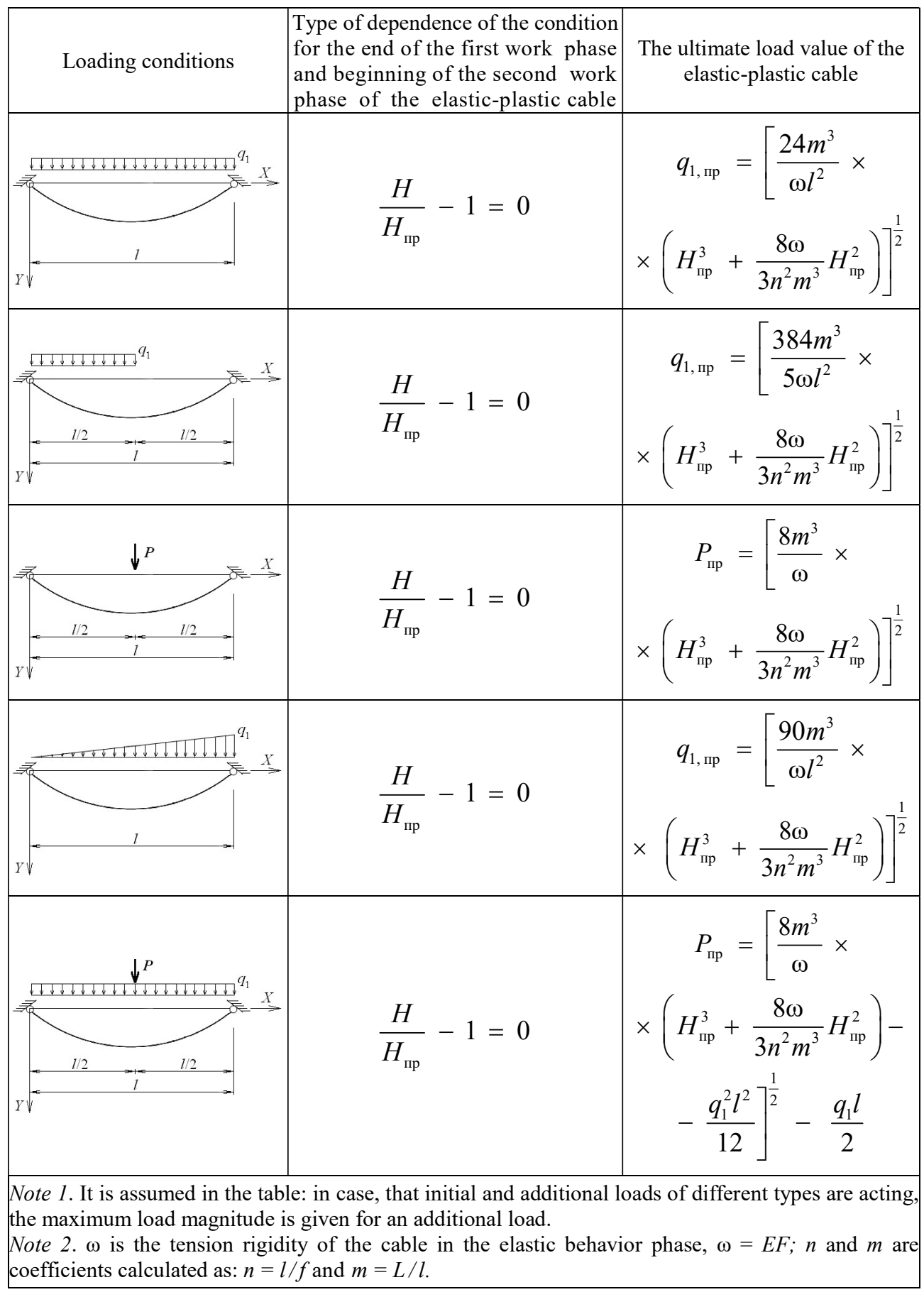




\section{Cables made of ideal elastic-plastic material with hardening}

Taking into account hardening in plastic deformation problems allows not only to consider the actual nature features of behavior of material, but also at the same time it makes it possible to reveal previously unreported structural strength reserves. In a number of practical calculation cases, these reserves can be large enough, so this refinement of $\sigma-\varepsilon$ diagram leads to more reliable solutions than those obtained by using an ideal elastic-plastic stretching diagram.

Let us supplement this with two more important remarks, the first of which concerns the general formulation of the problem. And its essence is this: the behavior of cables made of material with hardening can be considered using the assumptions and premises adopted in studying the work of perfectly plastic cables. But the second remark relates to general assembling sequence of the desired solution. It turns out that this sequence for the cable made of material with hardening does not differs practically from similar problems for elasticplastic cables. However, the solution, as a rule, is more complicated. Therefore, we consider here the simplest (after ideal plastic one) approximation of the material behavior diagram, namely: we take the material to be strengthened linearly (Fig. 1b).

\section{Cross-sectional behavior of cable made of ideal elastic-plastic material with hardening}

Let us consider the behavior of the cable cross section, regarding the material as elasticplastic with linear hardening. For this purpose, we turn to previously assembled equilibrium condition (1), and on its basis we analyze the behavior of an arbitrary cross section of the cable (Fig. 4a). Taking into account selected material model, we recall that stress $\sigma$ and deformation $\varepsilon$ in the elastic and elastic-plastic with strengthening ranges of behavior are interrelated by the condition given in the book [1]:

$$
\left.\begin{array}{ll}
\sigma=E \varepsilon & \text { with } \sigma \leq \sigma_{\mathrm{T}}, \\
\sigma=\sigma_{\mathrm{T}}\left(1-\frac{E_{0}}{E}\right)+E_{0} \varepsilon & \text { with } \sigma \geq \sigma_{\mathrm{T}},
\end{array}\right\}
$$

where $E_{0}$ is the material hardening modulus.

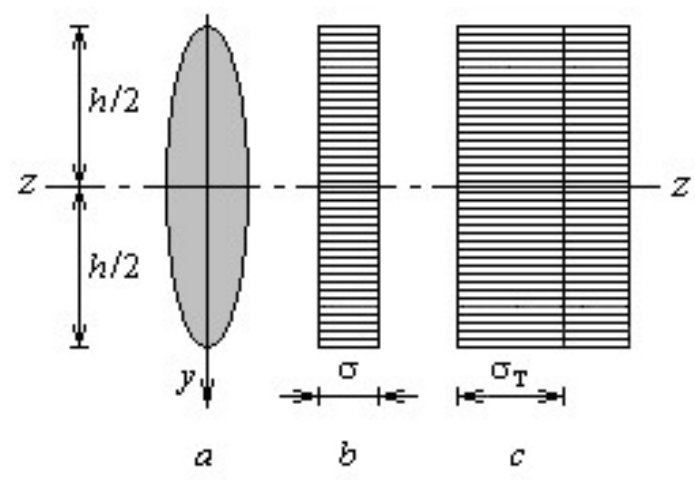

Fig. 4. The cross-sectional behavior of the cable made of elastic-plastic material with linear hardening: $a$ - cable cross-section; $b$ - stress diagram in the elastic range of behavior; $c$-stress diagram in the plastic range of behavior. 
Naturally, in this case it is necessary to consider two ranges of the cross section behavior: the first is the elastic range and the second is the plastic one. Let us note that the behavior of the cable cross section in the first range will be the same regardless of the material properties in the plastic sphere, and it was considered earlier in the monograph [9]. Therefore, we only recall here that the expression characterizing the condition for the end of the elastic range of the cross section behavior has the form (4).

Let us now turn to the second range of the cable cross section behavior, when the entire cross section is covered by plastic deformations (Fig. 4c). Substituting condition (5) into the equilibrium equation (1) we define the representation for the relative axis elongation of the cable $\varepsilon_{0}$ in the second behavior range in the following form:

$$
\frac{E \varepsilon_{0}}{\sigma_{\mathrm{T}}}=\frac{E}{E_{0}}\left(\frac{H}{H_{\text {пр }}}-1\right)+1 .
$$

Consequently, solution (6) completes the consideration of all possible behavior ranges for the cross-section of the cable made of elastic-plastic material with linear strengthening due to derivation of dependencies connecting the cross-sectional force acting in the cable with the relative elongation of its axis.

\section{Derivation of calculated dependences for cable made of ideal elastic-plastic material with hardening}

Based on the results obtained in the previous section, we establish the form of the calculated dependences of the refined deformation theory for elastic-plastic cables with linear hardening of material. Let us suppose that the cable with cross section $F$, as well as elastic modulus $E$ and linear hardening modulus $E_{0}$, is loaded with arbitrary vertical load $q(x)$ and is in equilibrium state (Fig. $5 a$ ).

We set the tension rigidity of the cable in the elastic behavior phase $\omega=E F$ and the plastic one $\omega_{0}=E_{0} F$ as an independent variable, and take the cross-sectional force $H$ as desired function.

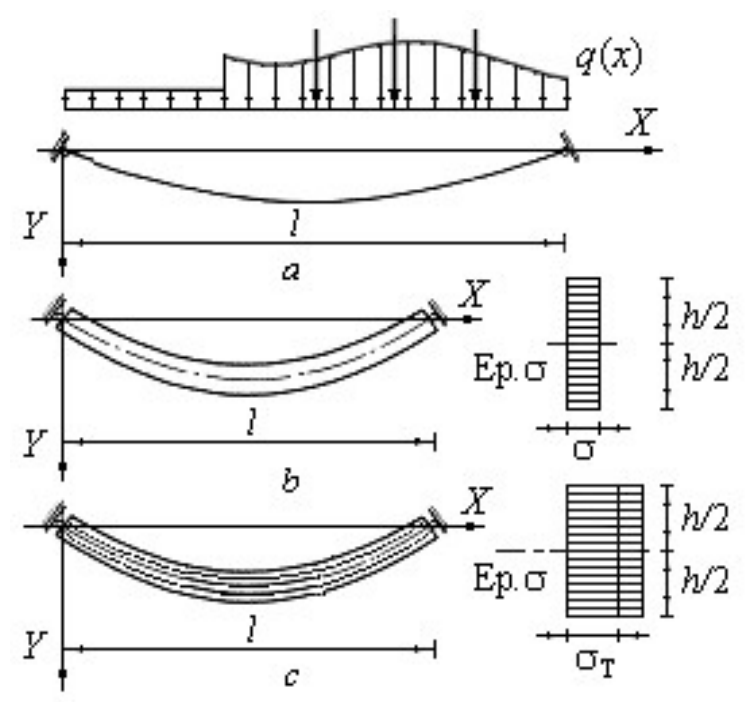

Fig. 5. Deformation of the cable made of elastic-plastic material with linear hardening: $a$ - design diagram of the cable; $b$ - elastic phase of behavior; $c$ - plastic phase of behavior with linear hardening of material. 
The behavior of the cable in the first deformation phase (Fig. $5 b$ ) when all its cross sections are behaving in elastic range is discussed in detail in the monograph [9]. Therefore, we shall not dwell on this here, but only recall that the elastic phase of behavior of the cable ends when condition (4) is satisfied and the magnitude of the cross-sectional force reaches its limit value.

When the external load reaches the value determined by equation (4), the second phase begins, the plastic phase of deformation, when the entire cable is covered by plastic deformations (Fig. $5 c$ ). We shall determine the calculated dependences during the behavior of the cable in this deformation phase. And for this purpose we turn to the principle of possible displacements.

In accordance with this principle we think that the stiffness $\omega_{0}$ obtained a very small but finite increment of its value equal to $\delta \omega_{0}$. Then the cross-sectional force $H$ will receive a certain increment $\delta H$, and the cable will move from its equilibrium position to the value $\delta y(x)$. In this case, the relations defining curved cable axis $y(x)$, the cable displacement $\delta y(x)$ and behavior of external forces $U_{1}$, and more precisely, the loads $q(x)$ on the displacements $\delta y(x)$, will still be of the form of:

$$
\begin{gathered}
y=\frac{M^{\sigma}}{H} ; \\
\delta y=\frac{M^{\sigma}}{H+\delta H}-\frac{M^{\sigma}}{H}=-\frac{M^{\sigma} \delta H}{H(H+\delta H)} \\
U_{1}=-\int_{0}^{l} \frac{q(x) M^{\sigma} \delta H}{H(H+\delta H)} d x=-\frac{D \delta H}{H(H+\delta H)} .
\end{gathered}
$$

Let us note that since these expressions are obtained using only equilibrium conditions, they are, of course, valid for any diagram $\sigma-\varepsilon$.

We define now the behavior of internal forces. In the second phase of behavior of the cable, the extension of its infinitesimal element $d s$ can be expressed as follows:

$$
\delta d s=\left(\frac{T+\delta T-T_{\text {пр }}}{\omega_{0}+\delta \omega_{0}}-\frac{T-T_{\text {пр }}}{\omega_{0}}\right) d s=\frac{\omega_{0} \delta T-T \delta \omega_{0}+T_{\text {пр }} \delta \omega_{0}}{\omega_{0}\left(\omega_{0}+\delta \omega_{0}\right)} d s .
$$

It is assumed here that $T$ is the longitudinal force in the cable, $\delta T$ is the increment in the magnitude of this force and $T_{\text {пр }}$ is the ultimate longitudinal force in the cable.

Elementary behavior of internal forces is defined as a product with the opposite sign of elongation $\delta d s$ by the amount of the force acting in the element, equal to $T+\delta T$, and the total behavior - as the integral of this product, extended over the entire cable length $L$ :

$$
U_{2}=-\int_{0}^{L} \frac{\left(\omega_{0} \delta T-T \delta \omega_{0}+T_{\text {пр }} \delta \omega_{0}\right)\left(T+\delta T-T_{\text {пр }}\right)}{\omega_{0}\left(\omega_{0}+\delta \omega_{0}\right)} d s .
$$

From geometric considerations, it is easy to understand that $T, T_{\text {пр }}, \delta T$, and $d s$ are expressed in terms of their projections onto the horizontal axis $X$ as follows:

$$
T=\frac{H}{\cos \alpha}, \quad T_{\text {пр }}=\frac{H_{\text {пр }}}{\cos \alpha}, \quad \delta T=\frac{\delta H}{\cos \alpha} \quad \text { and } \quad d s=\frac{d x}{\cos \alpha},
$$

where $\alpha$ is tangent inclination angle to the cable with respect to the horizon.

Now we have the opportunity to write out the full expression for behavior of internal forces:

$$
U_{2}=-\frac{\left(\omega_{0} \delta T-T \delta \omega_{0}+T_{\text {пр }} \delta \omega_{0}\right)\left(T+\delta T-T_{\text {пр }}\right)}{\omega_{0}\left(\omega_{0}+\delta \omega_{0}\right)} \int_{0}^{l} \frac{d x}{\cos ^{3} \alpha}
$$


The integral value in the last equation for gently sloping cables can be taken with sufficient accuracy to be equal to $l \mathrm{~m}^{3}$, where $1 / \mathrm{m}$ is the average value of $\cos \alpha$, equal to $l / L$. Then the expression for the behavior of internal forces can be finally represented as follows:

$$
U_{2}=-\frac{\left(\omega_{0} \delta H-H \delta \omega_{0}+H_{\text {пр }} \delta \omega_{0}\right)\left(H+\delta H-H_{\text {пр }}\right) l m^{3}}{\omega_{0}\left(\omega_{0}+\delta \omega_{0}\right)} .
$$

Let us now turn to the principle of possible displacements, according to which the sum of behavior of all external and internal forces on infinitesimal possible displacements of the system from the equilibrium position is equal to zero. Then successively substituting (9) and (14) into the condition $U_{1}+U_{2}=0$, then passing to the limit for $\delta \omega_{0} \rightarrow 0$ and $\delta H \rightarrow 0$, after some transformations we arrive at the following differential dependence between the values of the cross-sectional force and stiffness of the cable:

$$
\frac{d \omega_{0}}{d H}-\frac{\omega_{0}}{H-H_{\text {пр }}}-\frac{D \omega_{0}^{2}}{\operatorname{lm}^{3} H^{2}\left(H-H_{\text {пр }}\right)^{2}}=0 .
$$

This dependence is one of the special cases of the Bernoulli differential equation, whose general integral has the form:

$$
\frac{1}{\omega_{0}}=\frac{C}{H-H_{\text {пр }}}+\frac{D\left[H \ln H-H \ln \left(H-H_{\text {пр }}\right)-H_{\text {пр }}\right]}{l m^{3} H H_{\text {пр }}^{2}\left(H-H_{\text {пр }}\right)} .
$$

The value of an arbitrary integration constant $C$ can be found if we consider that in the case when the linear hardening modulus $E_{0}$ is equal to the modulus of elasticity $E$ (which also leads to the equality of the stiffness values $\omega_{0}$ and $\omega$ ), that is, when the cable is elastic, the cross-sectional force value $H_{1}$ can be determined from the well-known equation [9]:

$$
H^{3}+\frac{8 \omega}{3 n^{2} m^{3}} H^{2}=\frac{D \omega}{2 l m^{3}} .
$$

Substituting the value of the constant $C$ into expression (16), we arrive at the desired solution describing the behavior of elastic-plastic cables with linear hardening of material:

$$
\begin{gathered}
\frac{H-H_{\text {пр }}}{\omega_{0}}-\frac{H_{1}-H_{\text {пр }}}{\omega}=\frac{D}{l m^{3} H_{\text {пр }}^{2}}\left[\frac{H \ln H-H \ln \left(H-H_{\text {пр }}\right)-H_{\text {пр }}}{H}-\right. \\
\left.-\frac{H_{1} \ln H_{1}-H_{1} \ln \left(H_{1}-H_{\text {пр }}\right)-H_{\text {пр }}}{H_{1}}\right],
\end{gathered}
$$

and the value of $m$ is calculated here without taking into account elastic and plastic strains of the cable, that is, from its original geometry.

Thus, the behavior of elastic-plastic cable with linear strengthening of material is completely determined and the dependences for finding the parameters of the cable stressstrain state are found in all phases of its behavior.

\section{Investigation of behavior of cable made of ideal elastic-plastic material with hardening}

Let us now demonstrate application of the proposed calculation procedure for the example of the steel cable of ideal elastic-plastic material with linear hardening with the span of $l=$ $60 \mathrm{~m}$ with initial sagging deflection $f_{0}=4 \mathrm{~m}$, which corresponds to $1 / 15$ of the span, loaded with a uniformly distributed load throughout the whole span. The cable is assumed to have a 
circular cross section whose area is $F=1,2 \times 10^{-3} \mathrm{~m}^{2}$. The modulus of elasticity at behavior of material in elastic range is equal to $E=1,67 \times 10^{5} \mathrm{MPa}$, and the magnitude of the linear hardening modulus $E_{0}$ during material operation beyond the elastic limit varied from 1/10 to $1 / 40$ of the modulus of elasticity. The yield point of the material is assumed to be $\sigma_{\mathrm{T}}=335$ $\mathrm{MPa}$.

Let us define the behavior of a reinforced elastic-plastic cable under load. To do this, using the above expressions and calculated dependencies, the given values are calculated for displacements in the span middle of the cable and of the cross-sectional force in it at different ratios of strain hardening modulus and elasticity modulus $E_{0} / E$ for a number of values of a dimensionless intensity parameter of an uniformly distributed load $q l^{2} / M_{\text {пр }}\left(M_{\text {пр }}=\sigma_{\mathrm{T}} F d / 2\right.$, where $d$ is the diameter of the cable cross section), the curves of that are shown in Fig. 6.

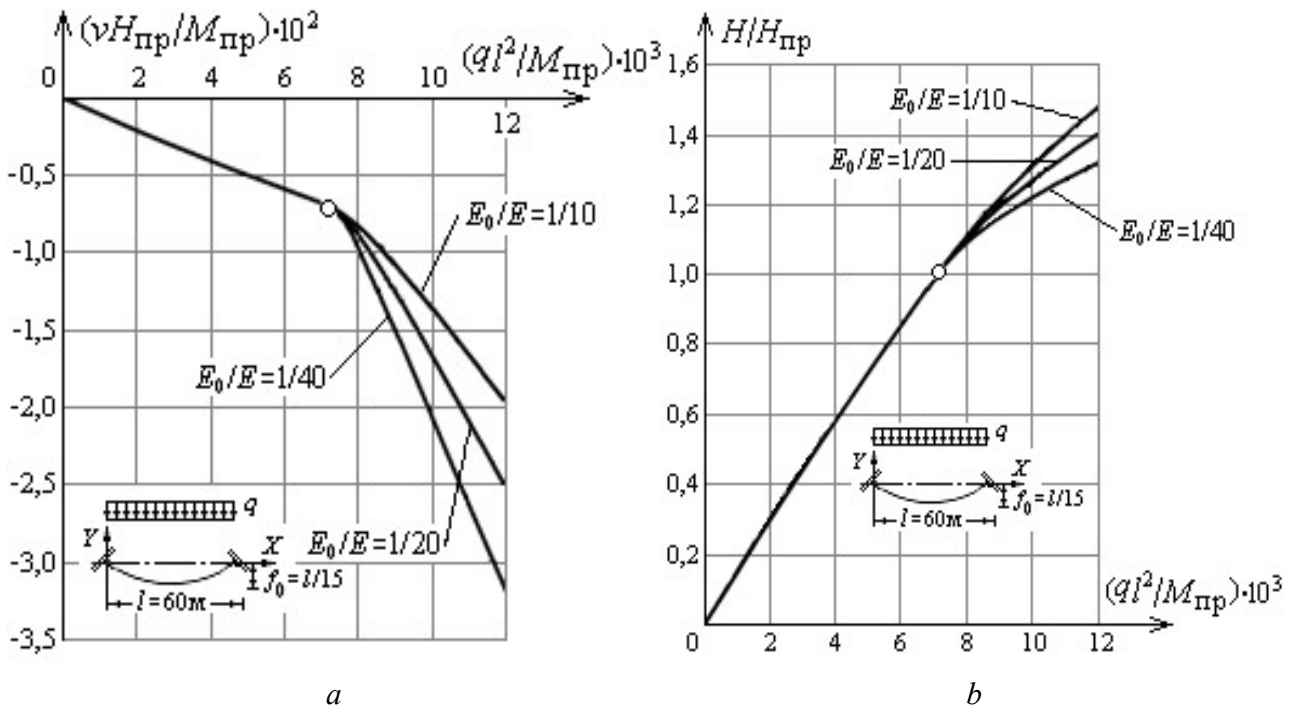

Fig. 6. Dependence between movements $(a)$ and cross-sectional force $(b)$ of elastic-plastic cable in the span middle from intensity of uniformly distributed load: $\circ$ - phase change of behavior of the cable.

As follows from analysis of obtained results and comparison of the curves in Fig. $6 a$, after transition of the cable to the plastic behavior phase with strengthening, its movements begin to increase sharply along the curve, the outline of which is close to the hyperbolic form. In this case, the smaller the material linear hardening module $E_{0}$, the faster the movement increases. It should also be noted that as the load increases, the displacement curves corresponding to the cable plastic behavior phase with hardening increasingly closer together. And with further increase in the load, the increment of displacements decreases.

From Fig. $6 b$ it can be seen that before the appearance of plastic deformations in the cable, the dependence of the cross-sectional force on acting load intensity is close to linear one. When the cross-sectional force $H$ reaches its limiting value of $H_{\text {пр }}$ and the cable becomes completely plastic, the break in curves occurs and the cross-sectional force in the cable begins to increase with the parabolic dependence as the load increases. In this case, the smaller the value of the material hardening modulus $E_{0}$, the slower the cross-sectional force expansion in the cable and the more sloping the corresponding curve is.

\section{References}


1. Н.И. Безухов, Основы теории упругости, пластичности и ползучести (Высшая школа, 1968) (N.I. Bezukhov, Fundamentals of the theory of elasticity, plasticity and creep)

2. Л.М. Беленький, Расчет судовых конструкциий в пластической стадии (Судостроение, 1983) (L.M. Belen'ky, Calculation of ship structures in the plastic range)

3. Дж. Н. Гудьер, Ф.Г. Ходж, Упругость и пластичность (Иностранная литература, 1960) (J.N. Goodier, F.G. Hodge, Elasticity and plasticity)

4. А.А. Ильюшин, Пластичность (Гостехиздат, 1948) (А.A. Ilyushin, Plasticity)

5. Л.М. Качанов, Основы теории пластичности (Гостехиздат, 1956) (L.M. Kachanov, Fundamentals of the theory of plasticity)

6. В.Т. Койтер, Общие теоремы теории упруго-пластических сред (Иностранная литература, 1961) (V.T. Koiter, General theorems of the theory of elastic-plastic media)

7. В. Прагер, Проблемы теории пластичности (Физматгиз, 1958) (V. Prager, Problems of the theory of plasticity)

8. В.В. Соколовский, Теория пластичности (Высшая школа, 1969). (V.V. Sokolovsky, Theory of plasticity)

9. А.В. Шимановский, В.В. Шалинский, Н.А. Чабан, Механика висячих систем (Сталь, 2013) (A.V. Shimanovsky, V.V. Shalinsky, N.A. Chaban, Mechanics of hanger systems) 